Linearization of a two-axis MEMS scanner driven by vertical comb-drive actuators

This article has been downloaded from IOPscience. Please scroll down to see the full text article.

2008 J. Micromech. Microeng. 18015015

(http://iopscience.iop.org/0960-1317/18/1/015015)

The Table of Contents and more related content is available

Download details:

IP Address: 140.112.113.225

The article was downloaded on 13/02/2009 at 07:04

Please note that terms and conditions apply. 


\title{
Linearization of a two-axis MEMS scanner driven by vertical comb-drive actuators
}

\author{
Jui-che Tsai ${ }^{1}$, Li-Cheng Lu ${ }^{1}$, Wei-Chi Hsu ${ }^{1}$, Chia-Wei Sun ${ }^{2}$ \\ and Ming $\mathrm{C} \mathrm{Wu^{3 }}$ \\ ${ }^{1}$ Graduate Institute of Photonics and Optoelectronics and Department of Electrical Engineering, \\ National Taiwan University, Taipei 10617, Taiwan \\ ${ }^{2}$ Medical Electronics and Device Technology Center, Industrial Technology Research Institute, Hsinchu, \\ Taiwan \\ ${ }^{3}$ Department of Electrical Engineering and Computer Sciences and Berkeley Sensor and Actuator Center \\ (BSAC), University of California, Berkeley, CA 94720-1774, USA
}

E-mail: jctsai@cc.ee.ntu.edu.tw

Received 2 August 2007, in final form 24 October 2007

Published 3 December 2007

Online at stacks.iop.org/JMM/18/015015

\begin{abstract}
A driving scheme using a pair of differential voltages $\left(V_{x}, V_{y}\right)$ over a bias voltage is proposed to linearize the dc characteristic (angle versus voltage) of a two-axis MEMS scanner. The micromirror has a gimbal-less structure and is driven by vertical comb-drive actuators in conjunction with a leverage mechanism. At an optimal bias voltage of $53 \mathrm{~V}$, a linear optical scan range of $\pm 3.2^{\circ}$ is achieved experimentally in both the $x$ and $y$ directions with the differential voltages ranging from $-10 \mathrm{~V}$ to $+10 \mathrm{~V}$.
\end{abstract}

(Some figures in this article are in colour only in the electronic version)

\section{Introduction}

MEMS (micro-electro-mechanical systems) scanners have been widely adopted in a variety of photonics-related research fields or products. In telecommunications, they are the key enabling components for two-dimensional (2D) [1] and three-dimensional (3D) [2, 3] optical cross-connects (OXCs), dynamic gain equalizers [4, 5], wavelength adddrop multiplexers (WADMs) [6, 7] and wavelength-selective switches (WSSs) [8-10]. They offer low optical insertion loss and crosstalk, independent of polarization and wavelength, as well as optical transparency for bit rate and data format. In adaptive optics, tip-tilt-piston micromirror arrays [11] and deformable mirrors [12] compensate wavefront distortions introduced by the medium and help achieve sharper images approaching the diffraction limit, which is particularly vital for space observation. MEMS optical scanners are also widely used in projection displays such as digital light processing [13] and laser scanning displays [14], endoscopic imaging [15] and confocal microscopy [16].

Electrostatic actuation is one of the most popular driving mechanisms for MEMS scanning mirrors [17]. Typically, capacitive structures are embedded to generate the required electrostatic torque for mirror rotation. The electrostatic torque, explicitly as a function of $V^{2}$, does not linearly vary with the actuation voltage. This results in strong distortion of the scan pattern when driving with linearly ramped voltages. In some cases, nonlinearity in the capacitance gradient with respect to rotation angle $(\theta)$ further aggravates the distortion; for instance, the capacitance of a parallel-plate scanner exhibits $1 / \theta$ dependence.

Several approaches have been proposed to eliminate the distortion, resulting in scan patterns with high linearity. Chiou et al divided the bottom electrode into multiple segments to fulfil the linearization of a one-axis parallel-plate micromirror [18]. Hiroshi et al demonstrated the linearization of a twoaxis parallel-plate MEMS scanner by using a differentialvoltage driving scheme, which was relatively simple and could be realized in open-loop operation [19]. The two polarities of the differential voltage $V_{\text {diff }}$ were superimposed on a bias voltage $V_{\text {bias. }}$ The resulting sum voltages, $\left(V_{\text {bias }}+V_{\text {diff }}\right)$ and $\left(V_{\text {bias }}-V_{\text {diff }}\right)$, were applied on the opposite electrodes. This induced electrostatic torques in opposite directions and proportional to $\left(V_{\text {bias }}+V_{\text {diff }}\right)^{2}$ and $\left(V_{\text {bias }}-V_{\text {diff }}\right)^{2}$, respectively, 

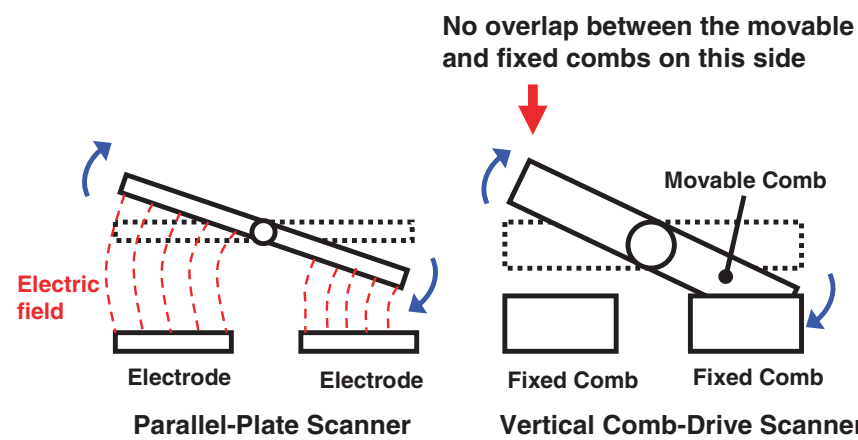
and fixed combs on this side

Figure 1. Comparison between parallel-plate and vertical comb-drive scanners.

on the mirror. The square terms $V_{\text {bias }}^{2}$ and $V_{\text {diff }}^{2}$ were cancelled out and only the term $V_{\text {bias }} \times V_{\text {diff }}$ remains in the net electrostatic torque, which is hence proportional to the control differential voltage $V_{\text {diff }}$ under a constant bias. Moreover, PID control (by Zhao et al [20]) and neural network methods (by Zhao et al [21]) have been respectively incorporated with the differential-voltage driving scheme to further enhance the scanner linearity.

The differential driving scheme performs best on the occasion that the capacitance derivative with respect to the rotation angle $(\partial \mathrm{C} / \partial \theta)$ remains constant, which unfortunately is not the case for parallel-plate scanners. On the other hand, vertical comb-drive actuators, whose $\partial \mathrm{C} / \partial \theta$ can be considered quasi-constant, seemingly prevail in this regard. However, the approach with differential-voltage driving is typically not suitable for rotating mirrors powered by vertical comb-drive actuators. This is due to the fact that, at the side that travels upward during rotation, the movable comb parts away from the fixed comb and their overlap vanishes at large angles. The consequence is that an opposite torque fails to be established, contrary to the case of parallel-plate scanners. This can be explained in figure 1 .

Recently, we have proposed a novel two-axis analog micromirror powered by vertical comb-drive actuators in conjunction with leverage mechanism [22] (figure 2). Large mechanical rotation angles $\left( \pm 6.7^{\circ}\right.$ for both axes at $\left.75 \mathrm{~V}\right)$

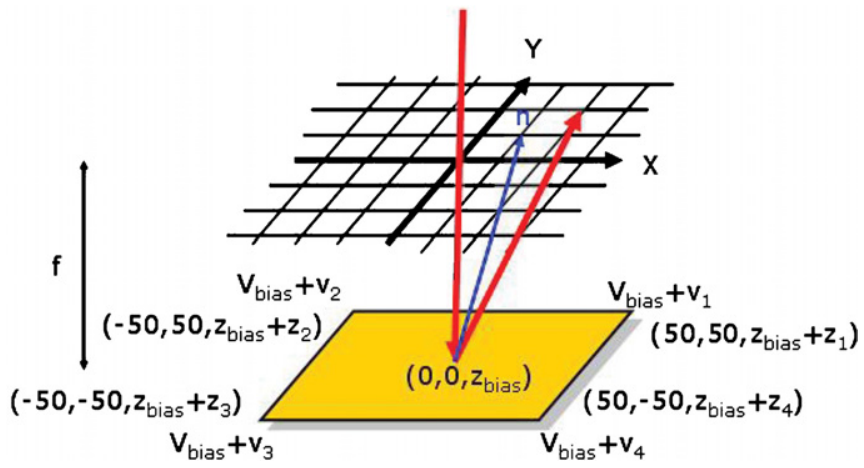

Figure 3. Simulation model. The unit for the numbers in this figure is $\mu \mathrm{m}$.

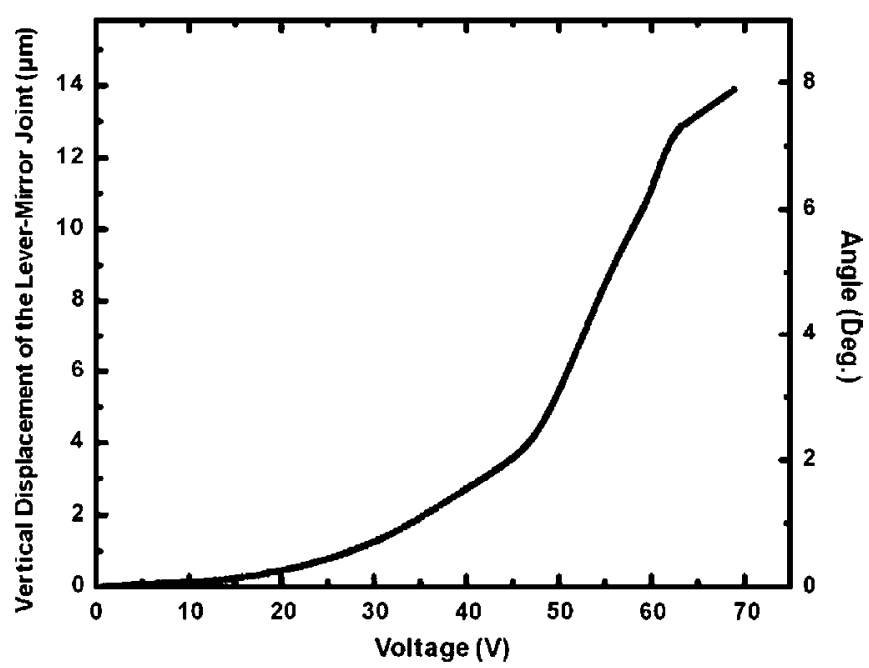

Figure 4. Lever characteristic: angle versus voltage, vertical displacement of the lever-mirror joint versus voltage.

and high resonant frequency $(5.9 \mathrm{kHz}$ before metallization) were achieved experimentally. The device was manufactured through a five-layer surface-micromachining process offered by Sandia National Laboratory. It can also be replicated along the $x$ direction to form a high fill-factor 1D mirror array [22].

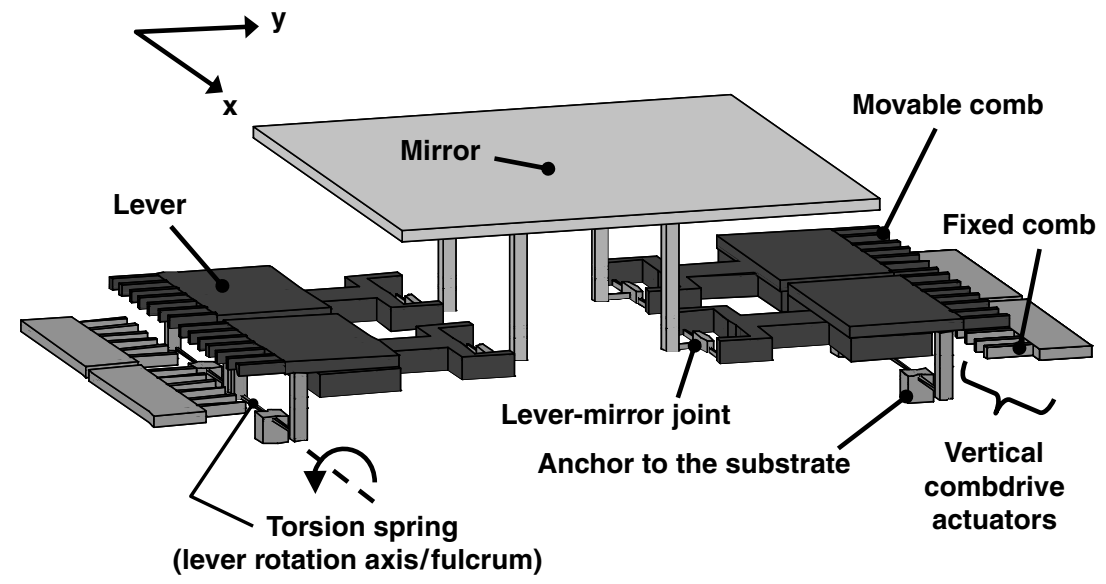

Figure 2. Schematic of the two-axis MEMS scanner driven by vertical comb-drive actuators in conjunction with a leverage mechanism [22]. 


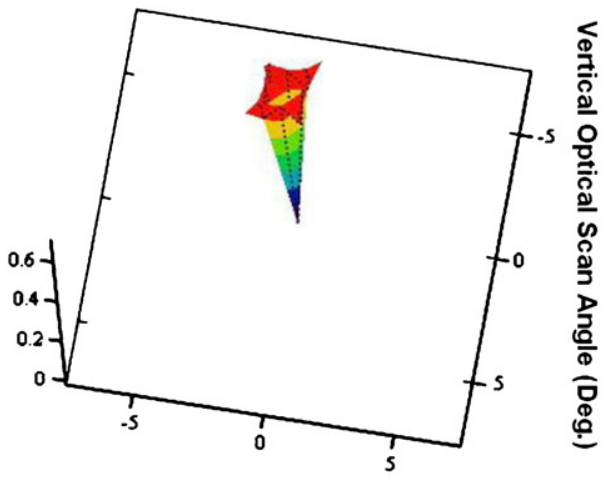

Horizontal Optical Scan Angle (Deg.)

(a)

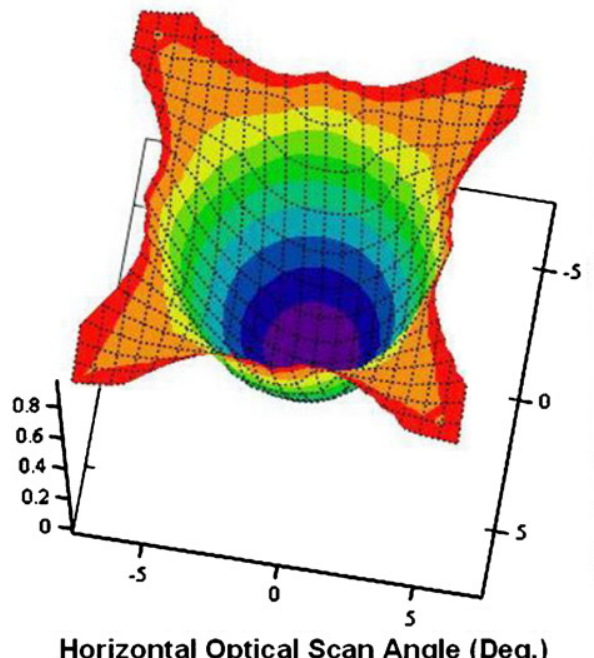

(c)

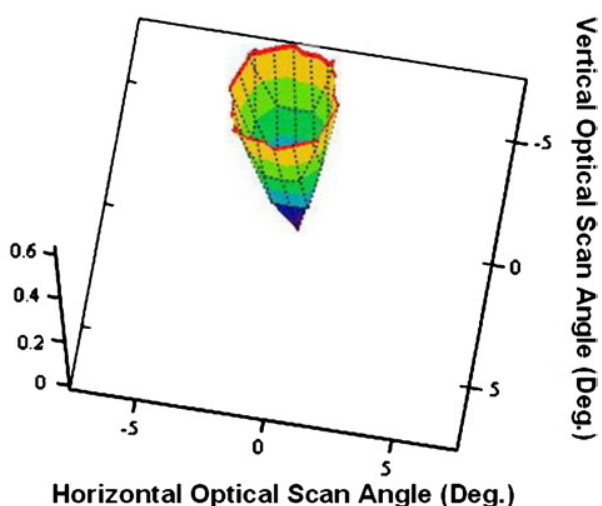

(b)

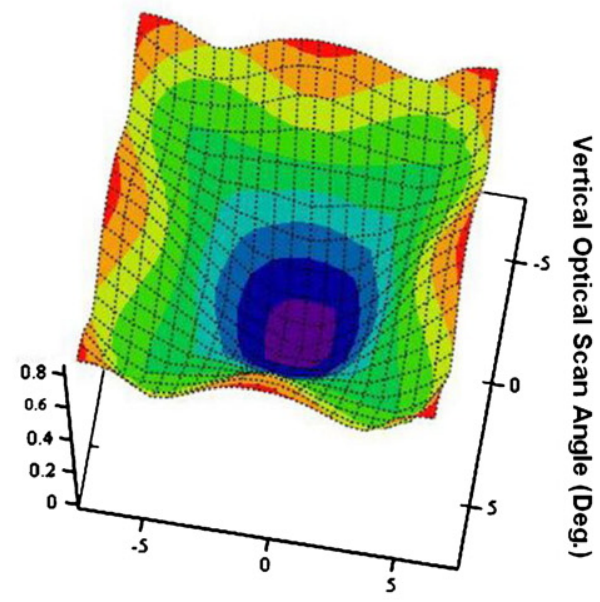

Horizontal Optical Scan Angle (Deg.)

(d)

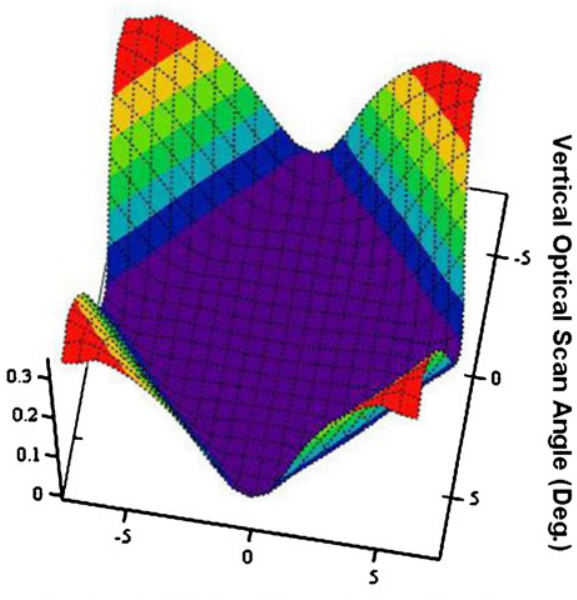

Horizontal Optical Scan Angle (Deg.)

(e)

Figure 5. Distribution of $E_{f, \text { total }}$ under different bias voltages: (a) $V_{\text {bias }}=20 \mathrm{~V}$, (b) $V_{\text {bias }}=30 \mathrm{~V}$, (c) $V_{\text {bias }}=40 \mathrm{~V}$, (d) $V_{\text {bias }}=50 \mathrm{~V}$ and (e) $V_{\text {bias }}=53 \mathrm{~V}$.

The mirror is equipped with four lever-comb-drive pairs and the design architecture ensures that all the four electrodes contribute toward the mirror rotation under any circumstance. Therefore, a differential driving scheme becomes possible for a comb-drive-driven mirror constructed in this manner. However, one has to note that the fundamental principle of differential driving of such a comb-drive-lever mirror is different from that of a parallel-plate scanner. As mentioned above, in the differential driving scheme of a parallel-plate mirror, electrostatic torques in the opposite directions are generated to cancel out the quadratic nonlinear terms. In our device, however, each comb-drive-powered lever possesses 


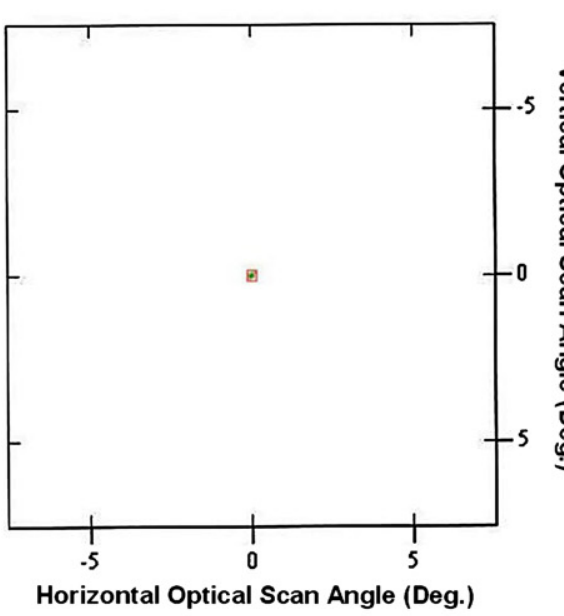

(a)

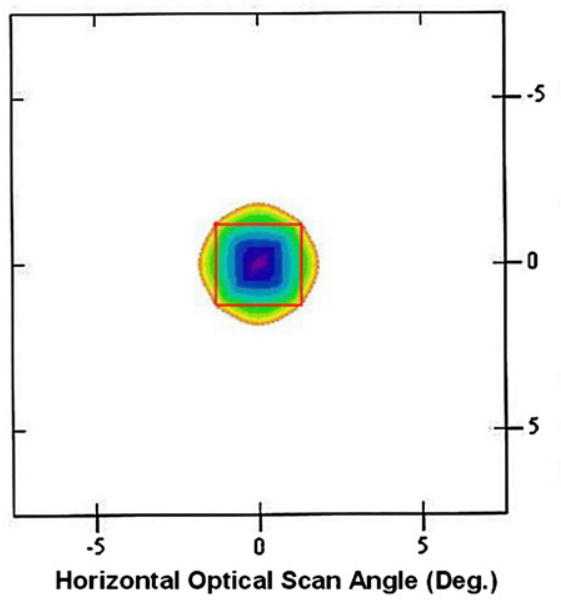

(c)

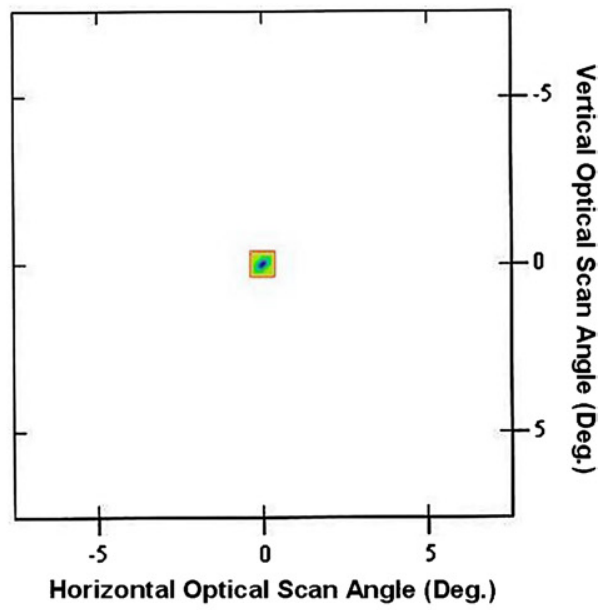

(b)

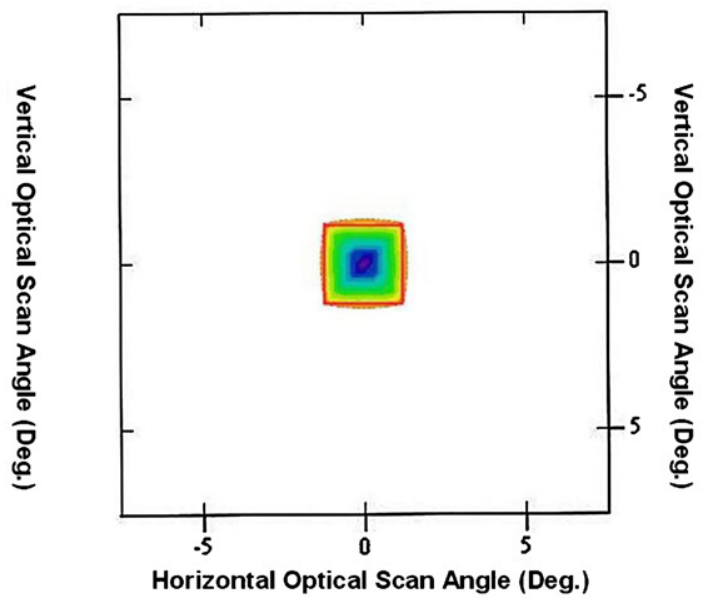

(d)

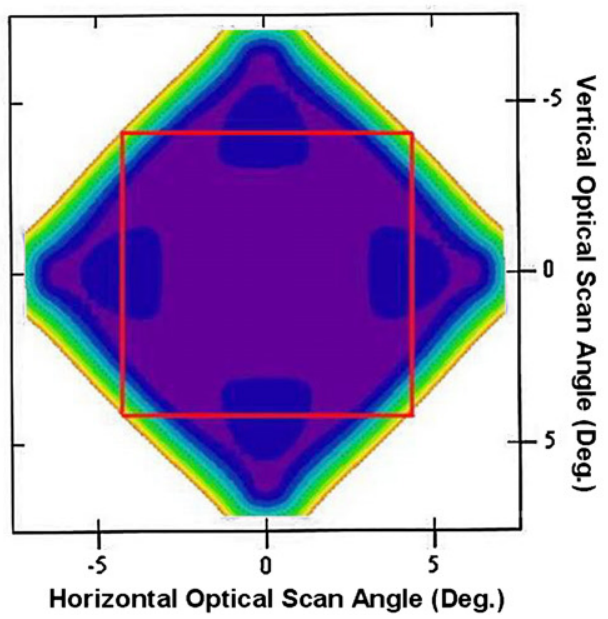

(e)

Figure 6. $x-y$ plane projections of $E_{f, \text { total }}$ for $(a) V_{\text {bias }}=20 \mathrm{~V}$, (b) $V_{\text {bias }}=30 \mathrm{~V},(c) V_{\text {bias }}=40 \mathrm{~V}$, $(d) V_{\text {bias }}=50 \mathrm{~V}$ and $(e) V_{\text {bias }}=53 \mathrm{~V}$, where the values greater than 0.1 are discarded. The red square is the largest square scan pattern that has its sides aligned parallel/orthogonally to the axes and is enclosed by the projection image.

control over the motion of one mirror corner. By measuring the angle-voltage characteristic of each lever, we are able to determine the optimal bias point to achieve the maximum scan pattern that exhibits high linearity using open-loop operation.

\section{Simulation determining the optimal bias point}

\subsection{Model}

At most four independent voltages can be allowed to operate our comb-drive-lever mirror. The voltages applied on the four 


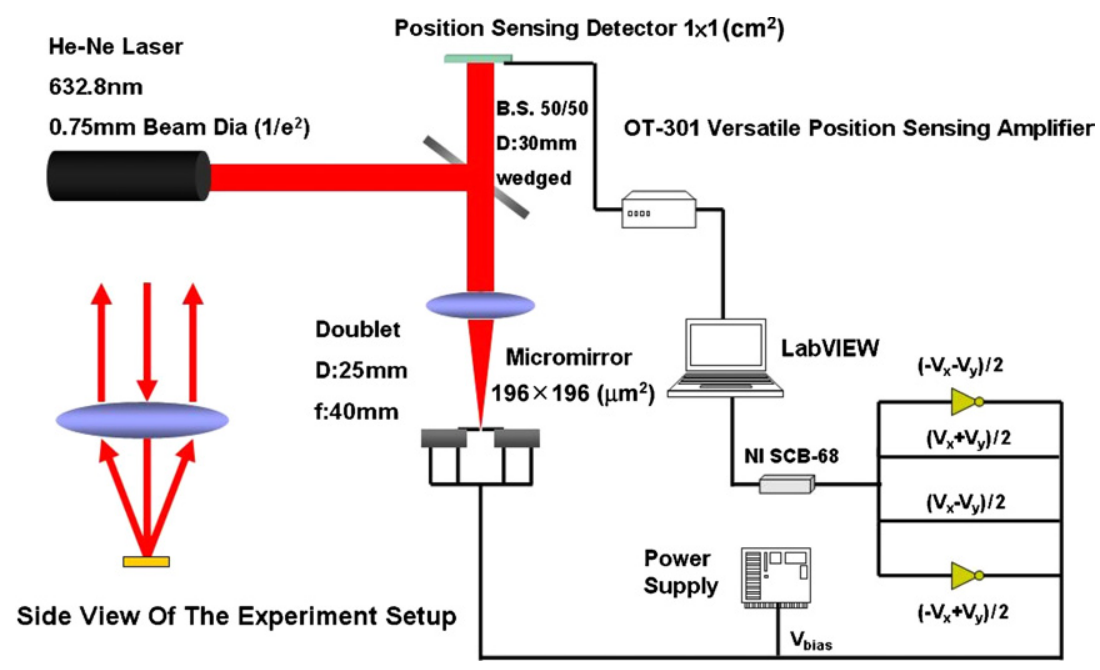

Figure 7. Schematic of the experimental setup.

levers are denoted as $V_{1}, V_{2}, V_{3}$ and $V_{4}$, respectively. We further express them as

$$
\begin{gathered}
V_{1}=V_{\text {bias }}+v_{1} \\
V_{2}=V_{\text {bias }}+v_{2} \\
V_{3}=V_{\text {bias }}+v_{3} \\
V_{4}=V_{\text {bias }}+v_{4} .
\end{gathered}
$$

In figure 3 , we define the coordinate of the mirror center as $\left(0,0, z_{\text {bias }}\right)$, where $z_{\text {bias }}$ represents the lift of the mirror center and is determined by the bias voltage $V_{\text {bias }}$. The coordinates of the four lever-mirror joints are $\left(50 \mu \mathrm{m}, 50 \mu \mathrm{m}, z_{\text {bias }}+z_{1}\right)$, $\left(-50 \mu \mathrm{m}, 50 \mu \mathrm{m}, z_{\text {bias }}+z_{2}\right),\left(-50 \mu \mathrm{m},-50 \mu \mathrm{m}, z_{\text {bias }}+\right.$ $\left.z_{3}\right)$, and $\left(50 \mu \mathrm{m},-50 \mu \mathrm{m}, z_{\text {bias }}+z_{4}\right)$, where $z_{1}, z_{2}, z_{3}$ and $z_{4}$ are determined by $v_{1}, v_{2}, v_{3}$ and $v_{4}$, respectively. $f$ is the distance between the mirror and the observation plane. For an incident light in the direction of $(0,0,-1)$ to be reflected to a point $(x, y)$ on the observation plane, the MEMS mirror has to be rotated so that its normal vector becomes $(x / 2, y / 2, f)$ as depicted in figure 3. For each targeted point on the observation plane, a combination of $\left(z_{1}, z_{2}, z_{3}, z_{4}\right)$ and a corresponding $\left(v_{1}\right.$, $v_{2}, v_{3}, v_{4}$ ) set can be solved. The step-by-step procedure is as follows:

(1) Experimentally measure the angle versus voltage curve of the lever and, hence, the vertical displacement of the lever-mirror joint versus voltage.

(2) Set a bias voltage $V_{\text {bias }}$ for all levers, which then determines $z_{\text {bias }}$ according to the curve obtained in step 1.

(3) Define the scan range and an array of targeted $(x, y)$ points on the observation plane.

(4) Find the mirror normal vector $n$ for the light to be reflected to a certain point $(x, y)$.

(5) For each $n$, find $z_{1}, z_{2}, z_{3}$ and $z_{4}$. Then the solution for $\left(v_{1}, v_{2}, v_{3}, v_{4}\right)$ can be obtained using the curve in step 1 along with $V_{\text {bias }}$ and $z_{\text {bias }}$. $\left(v_{1}, v_{2}, v_{3}, v_{4}\right)$ is considered as the control voltage set.
Table 1. Spans of the square patterns in figure 6: scan field size on the observation plane and the corresponding optical scan angle.

\begin{tabular}{lll}
\hline $\begin{array}{l}\text { Bias } \\
\text { voltage }(\mathrm{V})\end{array}$ & $\begin{array}{l}\text { Scan field size on the } \\
\text { observation plane }\left(\mu \mathrm{m}^{2}\right)\end{array}$ & $\begin{array}{l}\text { Optical scan angle in the } \\
x \text { and } y \text { directions }\left(^{\circ}\right)\end{array}$ \\
\hline 20 & $200 \times 200$ & \pm 0.14 \\
30 & $530 \times 530$ & \pm 0.38 \\
40 & $1800 \times 1800$ & \pm 1.3 \\
50 & $1670 \times 1670$ & \pm 1.2 \\
53 & $5900 \times 5900$ & \pm 4.22 \\
\hline
\end{tabular}

Generally fewer independent voltages are preferred as the control scheme can then be simplified. For a two-axis scanner, at least two independent voltages, typically denoted as $V_{x}$ and $V_{y}$, are required. Our purpose is therefore to reduce $\left(v_{1}, v_{2}\right.$, $\left.v_{3}, v_{4}\right)$ to $\left(V_{x}, V_{y}\right)$, i.e. to express $v_{1}, v_{2}, v_{3}$ and $v_{4}$ as linear combinations of $V_{x}$ and $V_{y}$ as follows:

$$
\begin{aligned}
& v_{1}=\frac{1}{2} \cdot\left(V_{x}+V_{y}\right) \\
& v_{2}=\frac{1}{2} \cdot\left(-V_{x}+V_{y}\right) \\
& v_{3}=\frac{1}{2} \cdot\left(-V_{x}-V_{y}\right) \\
& v_{4}=\frac{1}{2} \cdot\left(V_{x}-V_{y}\right) .
\end{aligned}
$$

At any given bias voltage, $V_{x}$ and $V_{y}$ can be solved only for the solutions in which $v_{1}=-v_{3}$ and $v_{2}=-v_{4}$. Therefore, we define the error functions as

$$
E_{f 1}=\left|\frac{\left|v_{2}\right|-\left|v_{4}\right|}{\left|v_{2}\right|+\left|v_{4}\right|}\right|, \quad E_{f 2}=\left|\frac{\left|v_{1}\right|-\left|v_{3}\right|}{\left|v_{1}\right|+\left|v_{3}\right|}\right|,
$$

and

$$
E_{f, \text { total }}=E_{f 1}+E_{f 2} .
$$

$E_{f 1}$ and $E_{f 2}$, each of which can actually be viewed as a ratio of the voltage difference to the average voltage, represent the degrees of $V_{2}-V_{4}$ and $V_{1}-V_{3}$ discrepancies, respectively. Ideally $E_{f \text {,total }}$ has to be 0 for exactly solving $V_{x}$ and $V_{y}$; however, we hereby define a tolerance 0.1 such that $V_{x}$ and $V_{y}$ are said to be 'quasi-solved' for $E_{f \text {,total }}<0.1$ (i.e., $10 \%) . \quad V_{x}$ and $V_{y}$ are then experimentally superimposed on the bias voltage $V_{\text {bias }}$ according to equations (1) and (2) so 


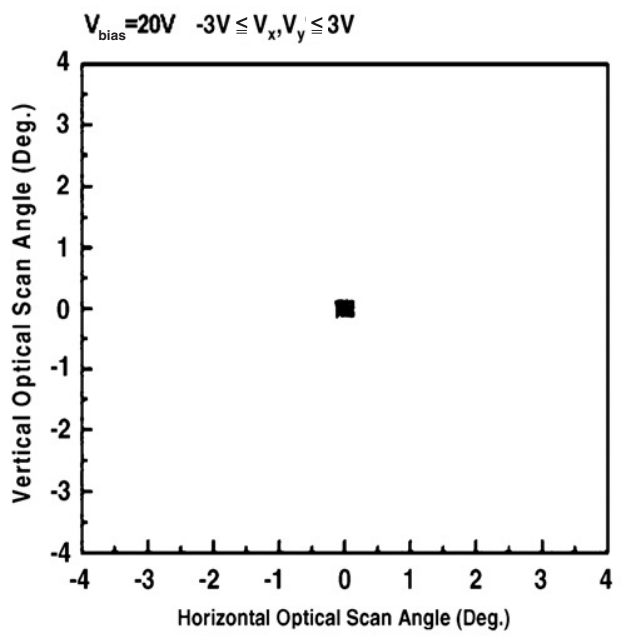

(a)

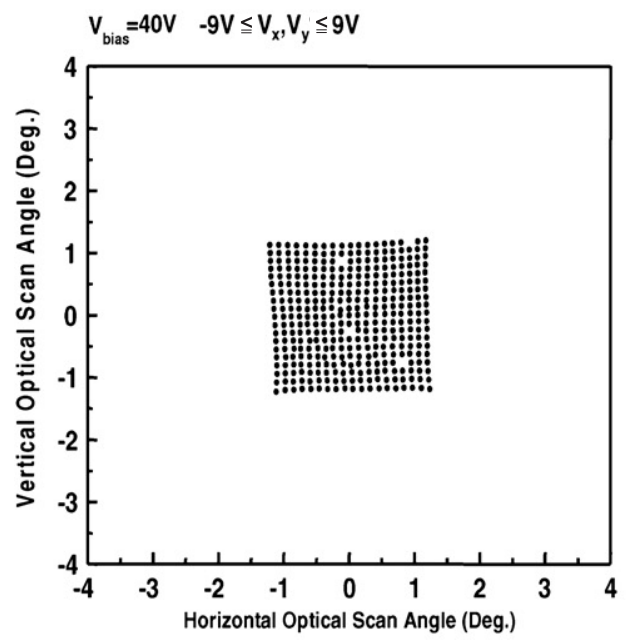

(c)

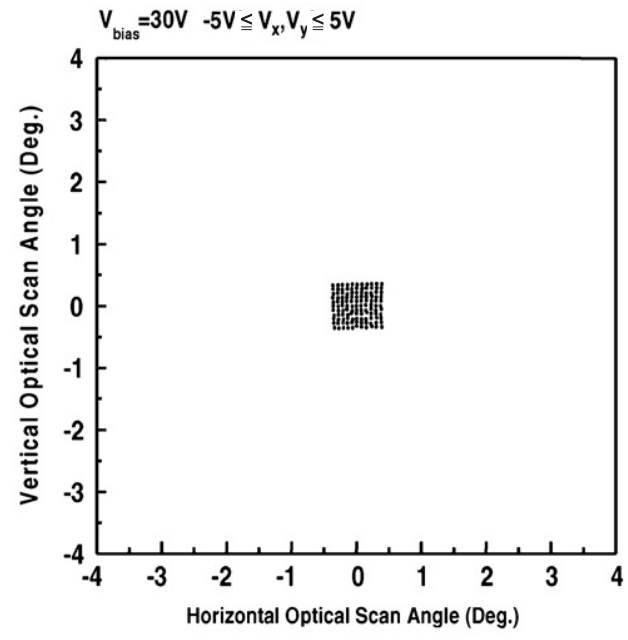

(b)

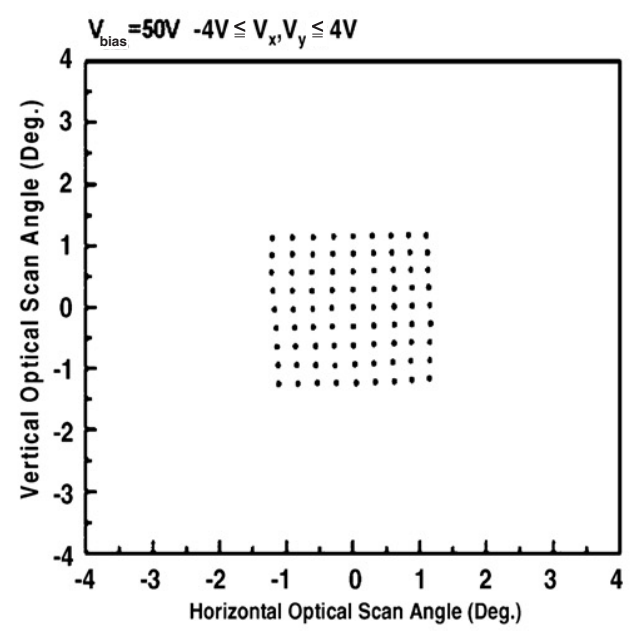

(d)

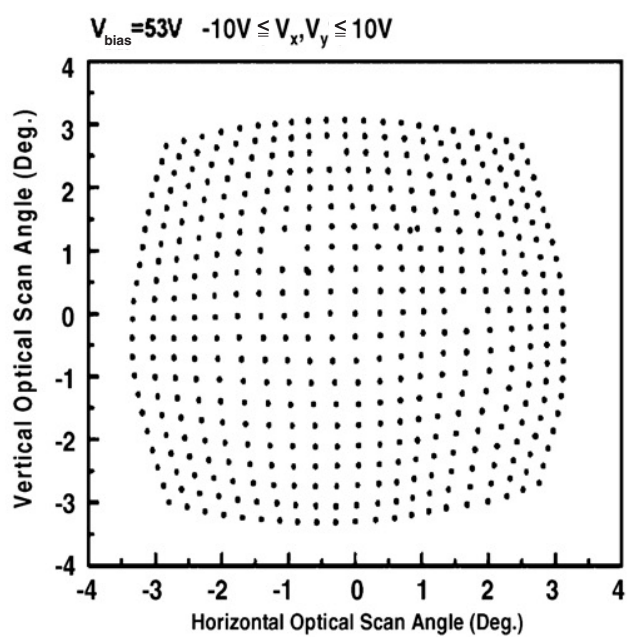

(e)

Figure 8. Scan fields measured by the PSD under different bias voltages: $(a) V_{\text {bias }}=20 \mathrm{~V},(b) V_{\text {bias }}=30 \mathrm{~V},(c) V_{\text {bias }}=40 \mathrm{~V}$, $(d) V_{\text {bias }}=50 \mathrm{~V}$ and $(e) V_{\text {bias }}=53 \mathrm{~V}$.

that a differential driving scheme can be achieved for two-axis rotation.

The coordinate axes in figures 2 and 3 are defined in a way that the $x$ and $y$ axes are parallel and orthogonal to the mirror array direction, respectively. This is mainly for the convenience of application and is adopted throughout the paper. However, it is worth noting that the coordinate axes can also be set along the mirror diagonals, i.e. $45^{\circ}$ rotation of the 


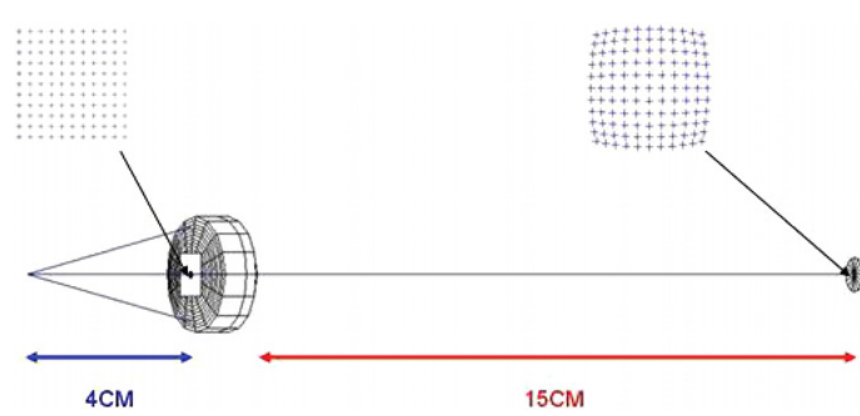

Figure 9. Effect of lens aberration on the measured scan pattern.

current coordinate system. In this case the lever-mirror joints are situated right on the axes. There is then no need to use the linear combinations of $V_{x}$ and $V_{y}$ in the differential driving scheme, i.e. they can be superimposed on the bias voltage separately.

\subsection{Simulation results}

Figure 4 is the measured individual lever characteristic. Simulations are done under various bias voltages. The distance between the mirror and the observation plane is set as $4 \mathrm{~cm}$, which is the focal length of the achromatic doublet lens used in the experiment. The experimental setup will be explained in the next section. The distributions of $E_{f \text {,total }}$ under different bias voltages, $20 \mathrm{~V}, 30 \mathrm{~V}, 40 \mathrm{~V}, 50 \mathrm{~V}$ and $53 \mathrm{~V}$, are plotted in figure 5. The $x-y$ plane projections of $E_{f \text {,total }}$ are shown in figure 6 , where the values greater than 0.1 are discarded. For each bias voltage, the largest square scan pattern that has its sides aligned parallel/orthogonally to the axes and is enclosed by the projection image is drawn with red solid lines. It is worth mentioning that at any given bias voltage some target points on the observation plane eventually yield solutions in which the resultant $z_{\text {bias }}+z_{i}(i=1,2,3$ or 4$)$ either goes beyond the maximum displacement in figure 4 or falls below 0 . Although these solutions have no physical meaning, the voltages $\left(v_{i}\right)$ and error-function values are nevertheless mathematically calculated by means of extrapolation on the curve in figure 4. After defining the square scan patterns in figure 6 , the solutions within the squares are rechecked to ensure that they do not fall within the aforementioned physically meaningless regime. Table 1 summarizes the spans of the square patterns in terms of the scan field size on the observation plane and the corresponding optical scan angle. It is concluded that the maximum scan range achievable by two independent control voltages occurs at $V_{\text {bias }}=53 \mathrm{~V}$. The optical scan range starts to decrease beyond this point. At $V_{\text {bias }}=60 \mathrm{~V}$ (not shown in the figures), it becomes $\pm 0.6^{\circ}$.

\section{Experiments}

\subsection{Differential driving operation}

A schematic of the experimental setup is shown in figure 7 . A $632.8 \mathrm{~nm} \mathrm{He}-\mathrm{Ne}$ laser is incident on the $50 / 50$ beam splitter with a $45^{\circ}$ incident angle. The laser beam $\left(1 / \mathrm{e}^{2}\right.$ beam diameter $2 w=0.75 \mathrm{~mm}$ ) is then focused onto the MEMS

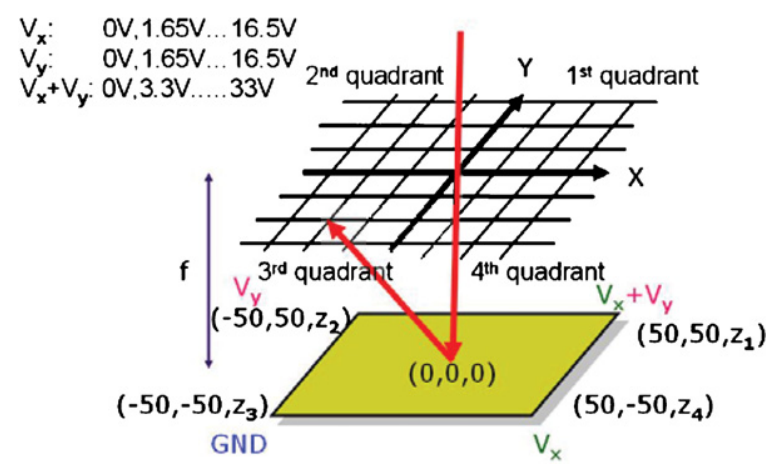

Figure 10. Non-differential driving scheme. The unit for the numbers in this figure is $\mu \mathrm{m}$.

scanner by an achromatic doublet lens with $4 \mathrm{~cm}$ focal length, resulting in a focus spot size of $2 w=43 \mu \mathrm{m}$. The differential voltages, $V_{x}$ and $V_{y}$, are computer programmed and the bias voltage is provided by a power supply. The MEMS scanner deflects the laser beam, which is then recollimated by the achromatic doublet lens, passes through the beam splitter, and finally is collected by the position-sensing detector (PSD). This arrangement ensures the scan pattern be independent of the distance between the PSD and the lens.

The differential voltages are varied with an increment of $1 \mathrm{~V}$ and are limited within $\pm 10 \mathrm{~V}$ by the data acquisition (DAQ) card. Figure 8 shows the scan fields experimentally measured by the PSD under different biases in the differentialdriving scheme. The scan range for $V_{\text {bias }}=53 \mathrm{~V}$ is limited to $\pm 3.2^{\circ}$ (in both the $x$ and $y$ directions) by the maximum voltage magnitude which can be provided by the DAQ card. It is expected that the value predicted by the simulation can be reached if sufficient driving voltage is supplied. We note that the required voltages to reach a certain angle in the experiment are larger than those in the simulation. This may result from the resistance at the lever-mirror joints, which are elastic and compliant, but actually not completely free.

The larger scan pattern for $V_{\text {bias }}=53 \mathrm{~V}$ exhibits distortion with a barrel shape, mainly caused by the lens aberration. The effect of lens aberration can be explained with the model in figure 9 constructed using ZEMAX, a commercial ray tracing software. A point source on the left focal plane emerging optical rays can be treated as the MEMS mirror steering the laser beam, where each ray is viewed as the reflected light beam from the mirror poised at a certain tilt angle. A $0.45 \mathrm{~cm} \times$ $0.45 \mathrm{~cm}$ square aperture, corresponding to the mirror scan pattern for $V_{\text {bias }}=53 \mathrm{~V}$, is placed right on the left of the lens. The transformed pattern at the PSD, $15 \mathrm{~cm}$ on the right of the lens, turns out to be a barrel shape due to the aberration of the achromatic doublet lens.

\subsection{Non-differential driving operation}

For comparison, a non-differential driving scheme with two independent voltages, $V_{x}$ and $V_{y}$, is implemented. The voltage combination shown in figure 10 deflects the laser beam toward the 3rd quadrant. In our experiment, $V_{x}$ and $V_{y}$ both vary from 0 to $16.5 \mathrm{~V}$ with an increment of $1.65 \mathrm{~V}$. This is achieved by 


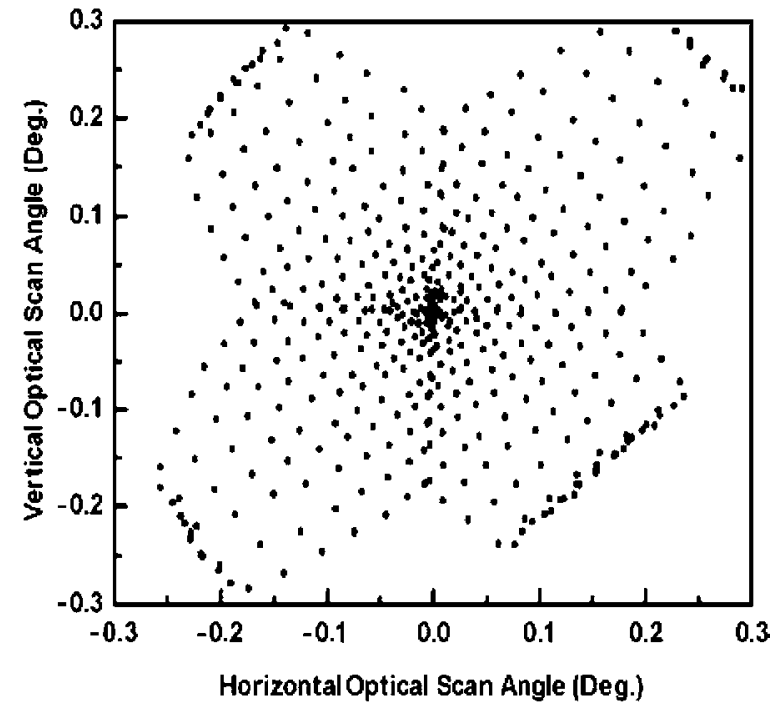

Figure 11. Scan field measured by the PSD under the non-differential driving scheme.

using power amplifiers for the voltages output by the computer. Similar voltage combinations can be used for beam deflection toward the 1st, 2nd and 4th quadrants. The complete scan pattern is shown in figure 11. It can be seen that the pattern is profoundly distorted even with a small optical scan range of $< \pm 0.3^{\circ}$. The slight asymmetry is due to optical system misalignment and the non-uniformity among levers.

\section{Conclusions}

We have implemented a differential driving scheme to linearize the dc characteristic of a two-axis MEMS scanner. The gimballess micromirror is driven by vertical comb-drive actuators in conjunction with leverage mechanism. A simulation model is developed to determine the maximum scan range. At an optimal bias voltage of $53 \mathrm{~V}$, a linear optical scan range of $\pm 3.2^{\circ}$ is achieved experimentally in both the $x$ and $y$ directions with the differential voltages varying within the range of $\pm 10 \mathrm{~V}$. It is expected that the value predicted by the simulation $\left( \pm 4.22^{\circ}\right)$ can be approached if sufficient driving voltage is supplied.

\section{Acknowledgments}

This work was supported by the National Science Council of Taiwan under grants NSC 95-2221-E-002-053 and NSC 96-2221-E-002-198-MY2, Excellent Research Projects of National Taiwan University, 95R0062-AE00-06, and DARPA/SPAWAR under contract N66001-00-C-8088.

\section{References}

[1] Fan L et al 2002 Digital MEMS switch for planar photonic crossconnects OFC 2002: Proc. Optical Fiber Communication (Anaheim, CA) Paper TuO4, pp 93-4

[2] Kozhevnikov M et al 2003 Compact $64 \times 64$ micromechanical optical cross connect IEEE Photon. Technol. Lett. 15 993-5
[3] Neilson D T et al $2004256 \times 256$ port optical crossconnect subsystem J. Light. Technol. 22 1499-509

[4] Goossen K W, Walker J A, Neilson D T, Ford J E and Knox W H 2000 Micromechanical gain slope compensator for spectrally linear optical power equalization IEEE Photon. Technol. Lett. 12 831-3

[5] Munechika M et al 2003 Optical dynamic gain equalizer using parallel motion type of ELCIA MEMS 2003: Proc. Micro Electro Mechanical Systems 2003 (Kyoto, 19-23 January) pp 243-6

[6] Ford J E, Walker J A, Aksyuk V and Bishop D J Wavelength selectable add/drop with tilting micromirrors Proc. IEEE Lasers and Electro-Optics Society Annual Meeting (San Francisco, CA, November 1997) Postdeadline paper PD2.3

[7] Ford J E, Aksyuk V A, Bishop D J and Walker J A 1999 Wavelength add-drop switching using tilting micromirrors J. Lightw. Technol. 17 904-11

[8] Marom D M et al 2005 Wavelength-selective $1 \times \mathrm{K}$ switches using free-space optics and MEMS micromirrors: theory, design, and implementation J. Light. Technol. 23 1620-30

[9] Tsai J C, Huang S, Hah D, Toshiyoshi H and Wu M C 2004 Open-loop operation of MEMS-based $1 \times \mathrm{N}$ wavelength-selective switch with long-term stability and repeatability IEEE Photon. Technol. Lett. 16 1041-3

[10] Tsai J C, Huang S, Hah D and Wu M C $20061 \times \mathrm{N}^{2}$ wavelength-selective switch with two cross-scanning one-axis analog micromirror arrays in a 4-f optical system J. Light. Technol. 24 897-903

[11] Dagel D J et al 2006 Large-stroke MEMS deformable mirrors for adaptive optics J. Microelectromech. Syst. 15 572-83

[12] Hishinuma Y and Yang E H 2004 Single-crystal silicon continuous membrane deformable mirror with PZT unimorph microactuator arrays Proc. Solid-State Sensor, Actuator and Microsystems Workshop (Hilton Head Island, SC, 6-10 June 2004) pp 87-90

[13] Hornbeck L J 1997 Digital light processing for high brightness, high resolution applications Proc. SPIE Electronic Imaging EI'97, Projection Displays III 3013 27-40

[14] Ko Y C et al 2006 Eye-type scanning mirror with dual vertical combs for laser display Sensors Actuators A 126 218-26

[15] Chong C et al 2006 Optically modulated MEMS scanning endoscope IEEE Photon. Technol. Lett. 18 133-5

[16] Ra H, Piyawattanametha W, Taguchi Y and Solgaard O 2006 Dual-axes confocal fluorescence microscopy with a two-dimensional MEMS scanner Proc. 2006 IEEE/LEOS Int. Conf. Optical MEMS and their Applications (Montana, USA, August 2006) pp 166-7

[17] Krishnamoorthy U, Lee D and Solgaard O 2003 Self-aligned vertical electrostatic combdrives for micromirror actuation IEEE/ASME J. Microelectromech. Syst. 12 458-64

[18] Chiou J C and Lin Y C 2003 A multiple electrostatic electrodes torsion micromirror device with linear stepping angle effect J. Microelectromech. Syst. 12 913-20

[19] Toshiyoshi H, Piyawattanametha W, Chan C T and Wu M C 2001 Linearization of electrostatically actuated surface micromachined 2-D optical scanner J. Microelectromech. Syst. 10 205-14

[20] Zhao Y, Tay F E H, Zhou G and Chau F S 2006 Fast and precise positioning of electrostatically actuated dual-axis micromirror by multi-loop digital control Sensors Actuators A 132 421-8

[21] Zhao Y, Tay F E H, Chau F S and Zhou G 2005 Linearization of the scanning field for 2D torsional micromirror by RBF neural network Sensors Actuators A 121 230-6

[22] Tsai J C and Wu M C 2006 Design, fabrication, and characterization of a high fill-factor, large scan-angle, two-axis scanner array driven by a leverage mechanism IEEE/ASME J. Microelectromech. Syst. 15 1209-13 